\title{
Electronic patient-reported outcomes: a revolutionary strategy in cancer care
}

\author{
Roland Eid¹, Fady GH Haddad ${ }^{1}$, Hampig Raphael Kourie ${ }^{*}, 1$ \& Joseph Kattan ${ }^{1}$ \\ ${ }^{1}$ Hematology-Oncology Department, Faculty of Medicine, Saint Joseph University, Beirut, Lebanon \\ * Author for correspondence: hampig.kourie@hotmail.com
}

First draft submitted: 30 July 2017; Accepted for publication: 23 August 2017; Published online: 10 November 2017

Keywords: advantages $\bullet$ editorial $\bullet$ electronic $\bullet$ impact $\bullet$ limitations $\bullet$ literature $\bullet$ patient-reported outcome $\bullet$ PRO - quality of life $\bullet$ survival

'ePROs' refers to 'electronic patient-reported outcomes'. But then again, what does it really mean? And what importance does it enclose?

A few years ago one could not hold a true discussion about this concept, not even with colleagues, due to the newness of this term, particularly in the oncology domain. Nowadays, oncologists are becoming more aware of the existence of such a strategy. However, are they really conscious of its weight and value?

As it has been known, PRO (patient-reported outcome) is a health outcome directly based on 'patient-self reporting'. It can cover a whole range of potential measurements, allowing the assessment of patient-reported health status for physical, mental and social wellbeing [1].

Today, medical technology and machines allow the measurement of many biochemical, physiological and physical data. However, they remain unable to collect other valuable data regarding the disease and its treatment, their consequent symptoms and adverse effects, the ensuing quality of life, etc. In fact, certain types of subjective data may only be obtained from the patient. For example, headaches, palpitations, nausea, fatigue, anxiety, depression or sleep disturbances are symptoms that are not always obvious to observers. Other examples are the frequency and severity of the symptoms, their impact on daily life and the perception or feeling of the patient toward the disease [2].

Different modalities have emerged in order to follow-up and assess the patients, starting from usual care, passing by paper-based PROs and getting to ePROS [3].

'Usual care' consists of patients discussing symptoms with physicians during clinic visits and calling their doctors when needed between visits. As its name implies, 'paper-based PROs' consists of patients reporting - their symptoms for instance - between visits, on a paper that they show to their physician during clinic visits. This means more patient-related data can be reported, including the detection of a bigger amount of complaints. A recent modality, which was found to be more efficient than paper based PROs, is the 'ePROs' or 'electronic PROs' [2].

In general, PROs strategies have the advantage of letting patients report more, express more and therefore be taken care of more [4]. In the ePROS, the outcomes of the patients are collected using electronic devices or software, such as the internet, computers, tablets, smartphones or downloadable applications. Patients can report during clinic visits and between visits. Moreover, real-time e-mail alerts can be sent to providers in case of a suspected alarming clinical deterioration, based on patient self-reporting symptoms. This explains one of the ePROs merits in oncology clinical care, which is their ability to enable regular and real-time monitoring of patient symptoms and needs, therefore enhancing the efficiency of care. Pain by itself can be better managed using a standardized ePROs method, such as questionnaires. Of course, the evaluation of a PRO instrument should take into account its validity, its reliability and its ability to detect change [5].

In this perspective, many advantages were described after the adoption of ePROs into routine cancer care, from improved patient-clinician communication to patient satisfaction and symptom management. Recently, improved health-related quality of life, less frequent admission to the emergency room or hospitalization, and longer tolerability of treatment (including chemotherapy) were also reported [6-8]. 
Apart from avoiding data entry errors and reducing missing information as compared with paper-based PRO, the most spectacular value of the ePROs consists in their ability, all by themselves, to increase the overall survival. This particular advantage will be highlighted later on [9].

The major barriers for the adoption of ePROs in our daily practice are logistical, due to the unfamiliarity of many patients with electronic devices. This is mainly related to their educational and socio-economical level, in addition to their technological skills. Moreover, the enthusiasm and the engagement of both clinicians and patients are mandatory to the good functioning of this treatment modality. The higher expense is another limiting factor; however, a cost-effectiveness study should be performed to determine the economic utility of this strategy [10].

On another scale, information obtained from PRO can also be used by regulatory authorities such as the US FDA. In fact, PRO assessment in reporting side effects led to a thoughtful incorporation of patients into clinical trials. PRO-Common Terminology Criteria for Adverse Events is a new tool developed by the FDA for assessing PRO side effects. The potential use of PRO measurement in early phase clinical trials may help in the evaluation of safety and dose optimization; in late phase trials, this may play a major role in the collection of informative adverse events. This method will probably prevent the under-reporting of these adverse events and may favor a better concordance of the results with those of real life $[11,12]$.

A recently published paper based on patient follow-up, showed an improvement in overall survival associated with the use of ePROS versus usual care in a randomized clinical trial. There was a gain of 5.2 months (31.2 vs 26 months) encountered in patients diagnosed with metastatic solid tumors receiving chemotherapy and assessed by ePRO for symptom monitoring during routine cancer treatment compared with usual care. In this study, 766 consecutive patients initiating routine chemotherapy were randomized either to the usual care group or to the PRO group, their median age was 66 years, $22 \%$ had less than a high school education and $30 \%$ were computer inexperienced. In the PRO group, patients provided self-report of 12 common symptoms from the National Cancer Institute's Common Terminology Criteria for Adverse Events via a web-based PRO questionnaire platform. The reported severe symptoms were transmitted to the clinical nurse via an e-mail alert and to the oncologist by a summary of symptoms burden at clinic visits [9].

It is very interesting to see that a new cancer-care management tool, based on PROs, can lead to an improvement of more than 5 months in overall survival compared with usual care. Can we continue to approve drugs that cost thousands of dollars in order to gain 1 or 2 months in progression-free survival, while nearly half a year of overall survival could be gained only by using a different cancer care modality, without adding any treatment? Thus, a directed cost-effectiveness study should be performed to determine the economic utility of this strategy in order to confirm that the real solution is in front of our hands and easy to obtain.

Financial \& competing interests disclosure

The authors have no relevant affiliations or financial involvement with any organization or entity with a financial interest in or financial conflict with the subject matter or materials discussed in the manuscript. This includes employment, consultancies, honoraria, stock ownership or options, expert testimony, grants or patents received or pending, or royalties.

No writing assistance was utilized in the production of this manuscript.

\section{References}

1. Basch E, Snyder C, McNiff K et al. Patient-reported outcome performance measures in oncology. J. Oncol. Pract. 10(3), 209-211 (2014).

2. Deshpande PR, Rajan S, Sudeepthi BL, Abdul Nazir CP. Patient-reported outcomes: a new era in clinical research. Perspect. Clin. Res. 2(4), 137-144 (2011).

3. Knoerl R, Gray E, Stricker C et al. Electronic versus paper-pencil methods for assessing chemotherapy-induced peripheral neuropathy. Support. Care Cancer doi: 10.1007/s00520-017-3764-y (2017) (Epub ahead of print).

4. Kotronoulas G, Kearney N, Maguire R et al. What is the value of the routine use of patient-reported outcome measures toward improvement of patient outcomes, processes of care, and health service outcomes in cancer care? A systematic review of controlled trials. J. Clin. Oncol. 32(14), 1480-1501 (2014).

5. Basch E. The rise of patient-reported outcomes in oncology. Presented at: ASCO Annual Meeting Chicago, IL, USA, 3-7 June 2016. https://am.asco.org/daily-news/rise-patient-reported-outcomes-oncology

6. Detmar SB, Muller MJ, Schornagel JH et al. Health-related quality-of-life assessments and patient-physician communication: a randomized controlled trial. JAMA 288(23), 3027-3034 (2002).

7. Velikova G, Booth L, Smith AB et al. Measuring quality of life in routine oncology practice improves communication and patient well-being: a randomized controlled trial. J. Clin. Oncol. 22(4), 714-724 (2004). 
8. Basch E, Deal AM, Kris MG et al. Symptom monitoring with patient-reported outcomes during routine cancer treatment: a randomized controlled trial. J. Clin. Oncol. 34(6), 557-565 (2016).

9. Basch E, Deal AM, Dueck AC et al. Overall survival results of a trial assessing patient-reported outcomes for symptom monitoring during routine cancer treatment. JAMA 318(2), 197-198 (2017).

10. Nelson EC, Eftimovska E, Lind C, Hager A, Wasson JH, Lindblad S. Patient reported outcome measures in practice. BMJ 350, g7818 (2015).

11. Willke RJ. Measuring the value of treatment to patients: patient-reported outcomes in drug development. Am. Health Drug Benefits 1(1), 34-40 (2008).

12. Kluetz PG, Chingos DT, Basch EM, Mitchell SA. Patient-reported outcomes in cancer clinical trials: measuring symptomatic adverse events with the National Cancer Institute's patient-reported outcomes version of the common terminology criteria for adverse events (PRO-CTCAE). Am. Soc. Clin. Oncol. Educ. Book 35, 67-73 (2016). 
